\title{
Heteromorphic bivalent association at meiosis in bread wheat
}

\author{
J. S. Heslop-Harrison, V. Chapman \\ and M. D. Bennett
}

\author{
Plant Breeding Institute, Maris Lane, Trumpington, \\ Cambridge, CB2 2LQ, England
}

\begin{abstract}
Crosses were made between 51 different pairs of ditelocentric lines of hexaploid bread wheat (Triticum aestivum L.) to give double-monotelodisomic progeny with two different telocentric chromosomes. All the crosses involved pairs of chromosomes from the same genome.

At meiosis, the two telocentric chromosomes paired with their normal homologous partners to form heteromorphic bivalents which could be distinguished from the other rod and ring bivalents at metaphase I-they were morphologically marked. The position which each marked bivalent occupied across the metaphase plate was scored in 5209 light-microscope equatorial squashes of pollen mother cells at metaphase I.

There was a strong tendency for the marked bivalents to be positioned towards, or at, the outside edge of the plate. This occurred in all the hybrid lines, and showed that morphologically marked bivalents do not behave like their normal homologues, at least in this hexaploid species. However, the crosses involving marked chromosomes in the $B$ genome tended to have the marked bivalents lying more often at the outside of the plate than did the crosses involving marked chromosomes from the $A$ and $D$ genome. Thus the genomes were behaving differently, and it is possible that the three genomes are spatially separated in the nucleus.
\end{abstract}

\section{INTRODUCTION}

The primary association of homologous chromosomes into pairs (bivalents) at the first metaphase of meiosis has been known during most of this century (see Darlington, 1937). Non-random, secondary associations of one or more bivalents into groups has also been noted by numerous authors since the 1930's (e.g., Darlington and Moffett, 1930) in several different polyploid plants, including Taraxacum species (Gustafsson, 1934), Oryza (Nandi, 1936) and Triticum aestivum (bread wheat; Riley, 1960; Kempanna and Riley, 1964; Feldman and Avivi, 1973).

Triticum aestivum is an allohexaploid species $(2 n=6 x=42)$ containing three different but genetically related genomes of various origins called $A, B$ and $D$ (Sears, 1952). It is impossible to identify most of the individual chromosomes of wheat in Feulgen-stained, light-microscope squashes at metaphase of mitosis or meiosis. Consequently, cells of stocks with known chromosomes marked as telocentrics, which can be distinguished from whole chromosomes at metaphase I of meiosis, were used both by Kempanna and Riley (1964) and in the present study. By using two marked chromosomes in various combinations, the relative spatial dispositions of pairs of different known marker chromosomes, present as telocentrics, or of heteromorphic bivalents, can be assessed.

Riley (1960) showed that within a metaphase I cell, two different cytologically marked, genetically related (homoeologous) bivalents, were immediately adjacent at metaphase I more frequently than would be the case if their positions were independent of each other. Later, more extensive experiments (Kempanna, 1963; Kempanna and Riley, 1964) demonstrated the secondary association of bivalents in bread wheat. These showed that two marked homoeologous bivalents were immediately adjacent more frequently than two non-homoeologues. They concluded that the secondary association depended upon genetic relationships between the associated bivalents. Besides showing the secondary association of homoeologues, the experiment indicated that different pairs of homoeologues may, on average, behave slightly differently. If so, this may be evidence for a pattern of bivalent positions within each genome-an order (as defined by HeslopHarrison and Bennett, 1983a) involving 
heterologues lying in fixed mean positions within genomes.

Kempanna and Riley looked at 14 crosses between the chromosomes belonging to two homoeologous groups, 1 and 7 , both within and between genomes. The main purpose of the present work was to extend these observations using a more complete sample ( 51 crosses) including only the crosses between different pairs of marker chromosomes from a single genome. The results were used to test for evidence of different behaviour of different pairs of marked bivalents within genomes in the wheat nucleus at metaphase I of meiosis which could relate to their relative spatial disposition. This study also investigates special, non-random, features relating to the positions of morphologically marked, as opposed to normal, unmarked, bivalents.

\section{MATERIALS AND METHODS}

The basic techniques were similar to those used by Kempanna (1963) and Kempanna and Riley (1964). The plants used were stocks derived from Triticum aestivum L. emend Thell. ssp. vulgare MacKey, cultivar Chinese Spring. Ditelocentric lines for each chromosome were intercrossed within each of the three genomes to give hybrid lines with different combinations of pairs of marked chromosomes. All combinations of pairs of chromosomes within each genome were made, except for the six involving chromosome $4 A$ and six with $4 D$. Thus, 21 double-monotelodisomic F1 lines were made in the $B$ genome, and 15 in each of the $A$ and $D$ genomes. The long chromosome arm was present as the telocentric except for $2 A$, $2 D, 6 A, 6 D$ and $7 D$ where short arms were present. Both long and short arms of chromosome $6 B$ (a nucleolus organising chromosome) were used in some of the crosses; as the results for each arm were not significantly different from each other, they are pooled below. The choice of male and female parent was made on the basis of seed or plant availability, plant fertility or arbitrarily.

The F1 plants resulting from the crosses were grown to meiosis in growth cabinets at $20^{\circ} \mathrm{C}$ with continuous illumination (irradiance about $110 \mathrm{~W} / \mathrm{m}^{2}$ ) except in a few cases in the $A$ and $D$ genomes where they were grown in a glasshouse. They were treated with non-systemic insecticides (Pyrethrum-based) when required, and fertilisers were given regularly.

Squashes of fixed anthers were made using standard techniques (Kempanna and Riley, 1964). Permanent slide preparations of pollen mother cells (PMCs) at metaphase I, either Feulgen, or propionic orcein stained, were made for cytological analysis. Slides within each set of crosses were coded to avoid bias through knowing which cross was being scored.

Only first metaphase cells with all 21 bivalents aligned along a straight or gently curved line (fig. 1) were selected for scoring. These were assumed to give flattened equatorial views of the metaphase plate. Except in a few cases where they occurred

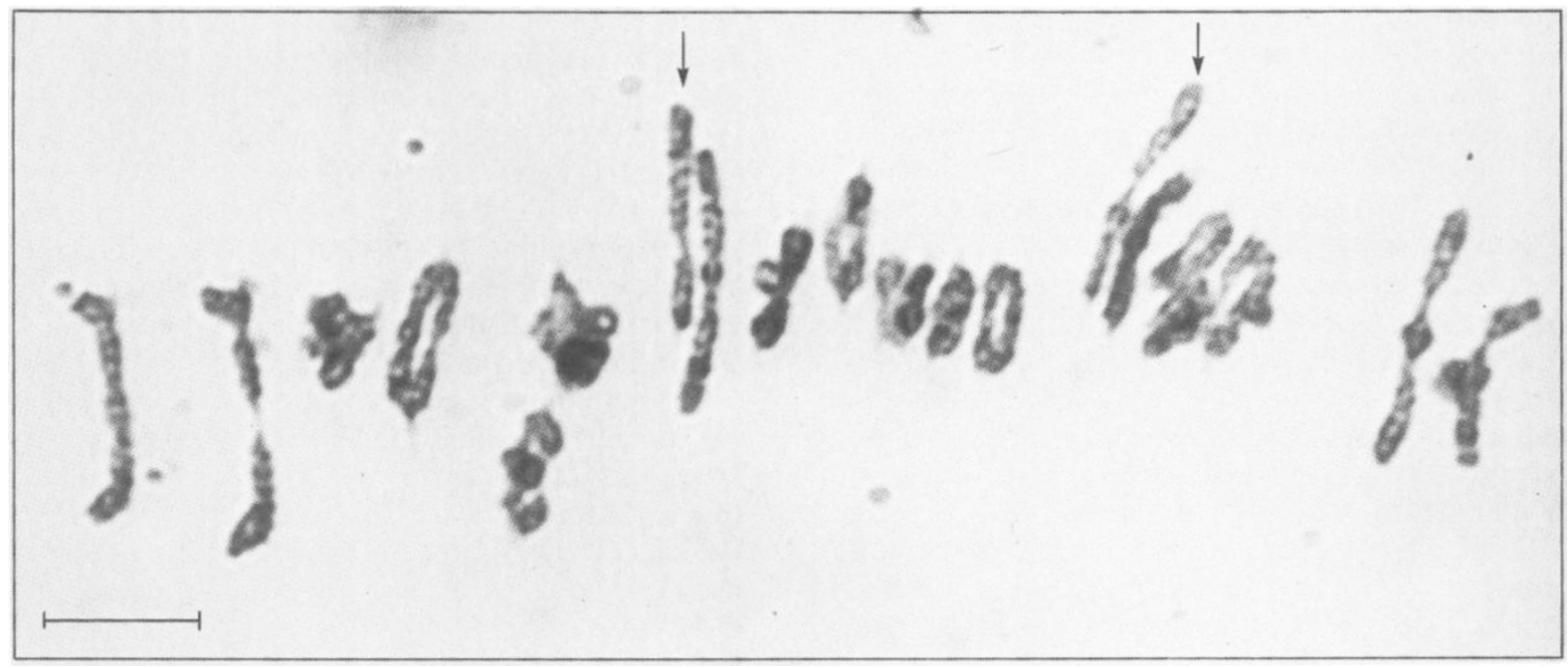

Figure 1 A metaphase plate typical of those scored from one of the crosses, involving $6 B$ and $7 B$ telocentric chromosomes. There are 19 normal bivalents (13 rings and 6 rods). The two heteromorphic bivalents (arrowed) are at positions 7 and 14 from the right end of the plate. (Scale bar $=10 \mu \mathrm{m}$.) 
as univalents, the telocentric and normal homologous partners paired at meiosis to form heteromorphic rod bivalents, which could be distinguished from normal bivalents at first metaphase (fig. 1). Slides were selected by a brief inspection at low power, before scoring, to choose those with a high proportion of well-spread pollen mother cells at metaphase I with bivalents in linear arrays.

A hundred or more cells at metaphase I were scored in each of the 51 hybrid lines. The positions of the heteromorphic marked bivalents on the metaphase plate were entered onto a scoring sheet which was rotated to be parallel with the linear array of the 21 bivalents. The scoring sheet consisted of a linear array of 21 boxes, numbered from left to right, to represent the bivalents in a PMC. The position of each of the two marked bivalents along the line of bivalents on the metaphase plate in a cell was indicated on the scoring sheet by an arrow in the corresponding box. If the two telocentric chromosome arms were towards the same spindle pole, the positions were indicated by two arrows pointing in the same direction. If they were towards different poles, the positions were indicated by arrows pointing in different directions.

As Kempanna and Riley (1964) noted, this scoring method was artificial in that the original three dimensional pattern of the metaphase plate was represented unidimensionally.

There is considerable evidence that the chromosome originally designated $4 B$ by Okamoto (1962) is chromosome $4 A$ (see Dvorak, 1983). Thus, analysis of the $B$ genome was carried out both with and without crosses including the chromosome designated $4 B$ by Sears. Since crosses involving chromosomes $4 A$ (as well as $4 D$ ) were not made, this consideration did not affect the analysis of the $A$ genome. In this paper, the chromosomes are referred to as originally named by Chapman and Riley (1966), following the work of Okamoto $(1962)$ and Sears $(1954 ; 1959)$.

\section{ANALYSES AND RESULTS}

\section{Directions of marked bivalents}

The data from the scoring sheets were entered into a microcomputer through a digitising tablet for analysis. Cells were divided into two groups, those with the two arrows (indicating the pole to which the telocentric chromosome would move) pointing in the same direction ( 2586 cells) and those with both pointing in different directions ( 2623 cells). The ratio of number of cells in these two groups was not significantly different from being equal $(P<0.001)$.

\section{Positions of marked bivalents}

Histograms were constructed from the data for positions of marked bivalents in each hybrid line. The number of occurrences of a marked bivalent at each of the 21 possible positions of bivalents across the plate was counted. Within the $A, B$ and $D$ genomes, $\mathrm{Chi}^{2}$ tests of heterogeneity showed no significant variation between the hybrid lines ( $A$ genome $\mathrm{Chi}^{2}=273(P>0 \cdot 1) ; B$ genome $\mathrm{Chi}^{2}=$ $442(0 \cdot 1>P>0 \cdot 05) ; D$ genome $\mathrm{Chi}^{2}=296(P>$ $0 \cdot 1)$ ). Consequently, the results were pooled within genomes.

Because of the symmetry of the metaphase plate, the number of marked bivalents at the two ends of the plate (positions 1 and 21) were summed into the first column of the histogram, those one in from each end (positions 2 and 20) were summed into the second column, etc. until the central position, 11 from each end of the plate, was reached, where the number of marked bivalents actually occurring was plotted. Thus, the first 10 columns of the histogram each included the marked bivalents occurring at two positions, while position eleven contained those occurring at only one position. Fig. 2(i), (ii) and (iii) shows histograms of bivalent positions for all the cells which were scored in each of the three genomes. The first position, giving the number of marked bivalents occurring at the outside or periphery of the flattened metaphase plate, will be referred to as the "end", while position 11 will be referred to as the "centre".

If marked bivalents occurred randomly over the metaphase plate, equal numbers would be expected in each of the 21 possible positions. The three distributions actually found were very highly significantly different (see table $1 ; P$ less than $0 \cdot 001$ in all three genomes) from this random expectation (shown as a broken line in fig. 2). For all genomes, marked bivalents tended to lie towards the end of the metaphase plate. Genome $B$ was significantly different from the other two genomes $\left(\mathrm{Chi}^{2}=\right.$ 106.8, $A$ genome and 107.5, $D$ genome; $P<$ $0.001)$. In the $B$ genome, marked bivalents tended to lie at the extreme end of the plate. The $A$ and $D$ genomes, which were not significantly different from each other $\left(\mathrm{Chi}^{2}=6 \cdot 0\right.$, N.S. $)$, showed a tendency for the marked bivalents to lie near, but not at, the end position of the metaphase plate. The $B$ genome without the six crosses involving bivalent $4 B$ as a heteromorph was not significantly different 


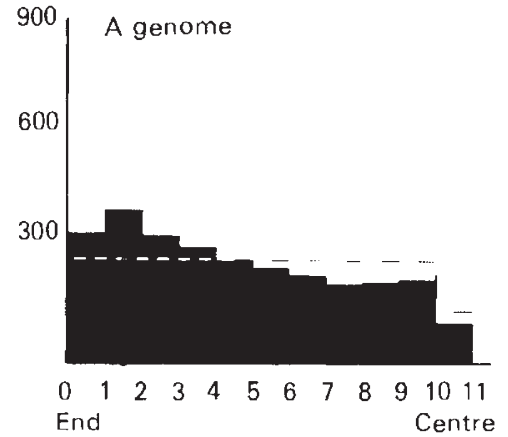

(i)

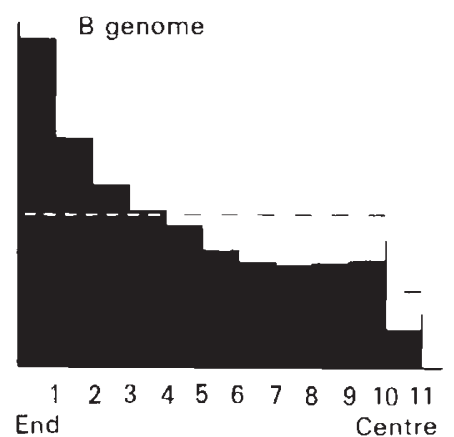

(ii)

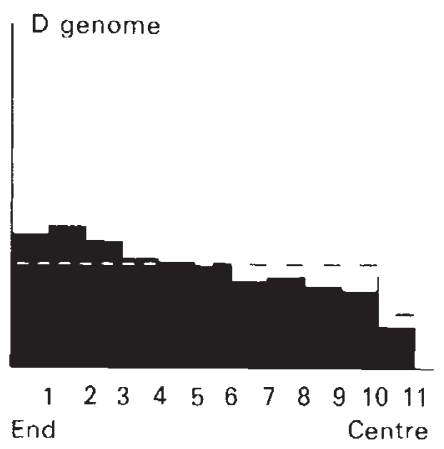

(iii)

Figure 2 Histograms for each of the three genomes showing the distribution of the positions of the marked bivalents across the linear metaphase plates. The expected distribution if marked bivalents were positioned at random, a straight line, is shown as a broken line on each graph. (a) 1,505 $\mathrm{A}$ genome crosses. (b) 2,204 $\mathrm{B}$ genome crosses. (c) $1,500 \mathrm{D}$ genome crosses. All three are significantly different from the random distribution, and (b) is highly significantly different from distributions (a) and (c).

Table 1 Comparison of the number of marked bivalents occurring at each position across the linear metaphase plate. If distributed at random, bivalents would occur at positions 1 to 10 with equal frequencies, and position 11 with half that frequency (see text). (Abbreviations: No $=$ Number, Ex $=$ Expectation, N.S. $=$ Not Significant, ${ }^{*}=$ significant at 5 per cent level, $\dagger=s i g n i f i c a n t$ at 1 per cent, $\ddagger=$ significant at 0.1 per cent; these abbreviations are also used in the text.) Numbers may not add to totals indicated because of rounding in this and other tables

\begin{tabular}{|c|c|c|c|c|c|c|c|c|c|}
\hline \multirow[b]{2}{*}{ Position } & \multicolumn{3}{|c|}{ A } & \multicolumn{3}{|c|}{$\begin{array}{c}\text { Genome } \\
B\end{array}$} & \multicolumn{3}{|c|}{$D$} \\
\hline & No & Ex & $\mathrm{Chi}^{2}$ & No & Ex & $\mathrm{Chi}^{2}$ & No & Ex & $\mathrm{Chi}^{2}$ \\
\hline 1 & 357 & 287 & $17 \cdot 3$ & 898 & 420 & $544 \cdot 7$ & 369 & 286 & $24 \cdot 3$ \\
\hline 2 & 421 & 287 & $63 \cdot 0$ & 631 & 420 & $106 \cdot 2$ & 391 & 286 & $38 \cdot 8$ \\
\hline 3 & 354 & 287 & $15 \cdot 8$ & 498 & 420 & $14 \cdot 6$ & 347 & 286 & $13 \cdot 2$ \\
\hline 4 & 323 & 287 & $4 \cdot 6$ & 427 & 420 & 0.1 & 300 & 286 & $0 \cdot 7$ \\
\hline 5 & 282 & 287 & 0.1 & 388 & 420 & $2 \cdot 4$ & 290 & 286 & 0.1 \\
\hline 6 & 264 & 287 & $1 \cdot 8$ & 321 & 420 & $23 \cdot 3$ & 276 & 286 & $0 \cdot 3$ \\
\hline 7 & 236 & 287 & $9 \cdot 0$ & 293 & 420 & $38 \cdot 3$ & 244 & 286 & $6 \cdot 1$ \\
\hline 8 & 215 & 287 & $17 \cdot 9$ & 279 & 420 & $47 \cdot 2$ & 248 & 286 & $5 \cdot 0$ \\
\hline 9 & 219 & 287 & $16 \cdot 0$ & 280 & 420 & $46 \cdot 6$ & 220 & 286 & $15 \cdot 1$ \\
\hline 10 & 229 & 287 & $11 \cdot 6$ & 293 & 420 & $38 \cdot 3$ & 210 & 286 & $20 \cdot 1$ \\
\hline 11 & 110 & 143 & $7 \cdot 8$ & 100 & 210 & $57 \cdot 6$ & 105 & 143 & $10 \cdot 0$ \\
\hline $\begin{array}{l}\text { Totals } \\
\text { Significance }\end{array}$ & 3010 & & $\begin{array}{l}164 \cdot 7 \\
(\ddagger)\end{array}$ & 4408 & & $\begin{array}{l}919 \cdot 2 \\
(\$)\end{array}$ & 3000 & & $\begin{array}{l}133 \cdot 6 \\
(\ddagger)\end{array}$ \\
\hline
\end{tabular}

from the six crosses including this marked bivalent $\left(\mathrm{Chi}^{2}=0 \cdot 7\right.$, N.S. $)$.

\section{Separations of marked bivalents}

A second type of histogram was constructed (fig. 3) which showed the frequency of occurrence of different numbers of intervening bivalents between the two marked bivalents in the sample of pollen mother cells scored. If the two marked bivalents in one cell were adjacent, then the number of intervening bivalents was 0 ; if they were at opposite ends of the plate, the separation was 19. These histograms are broadly similar to the graphs shown by Kempanna and Riley (1964) for nonhomoeologous bivalent association. Indeed, the graphs for the $A$ and $D$ genomes were not significantly different from those given by the earlier authors $\left(\mathrm{Chi}^{2}=17.9(A\right.$ genome, $0.7>P>0.5)$ and $28.2(D$ genome, $0 \cdot 1>P>0.05)$ ), although the $B$ genome was significantly different $\left(\mathrm{Chi}^{2}=\right.$ $42 \cdot 5, P<0 \cdot 01$ ).

Table 2 shows the separation of pairs of marked bivalents in the three genomes, with the expectation if the marked bivalents were distributed at random across the unidimensional metaphase plate. The most significant differences between genomes occur in the classes with no interveners 

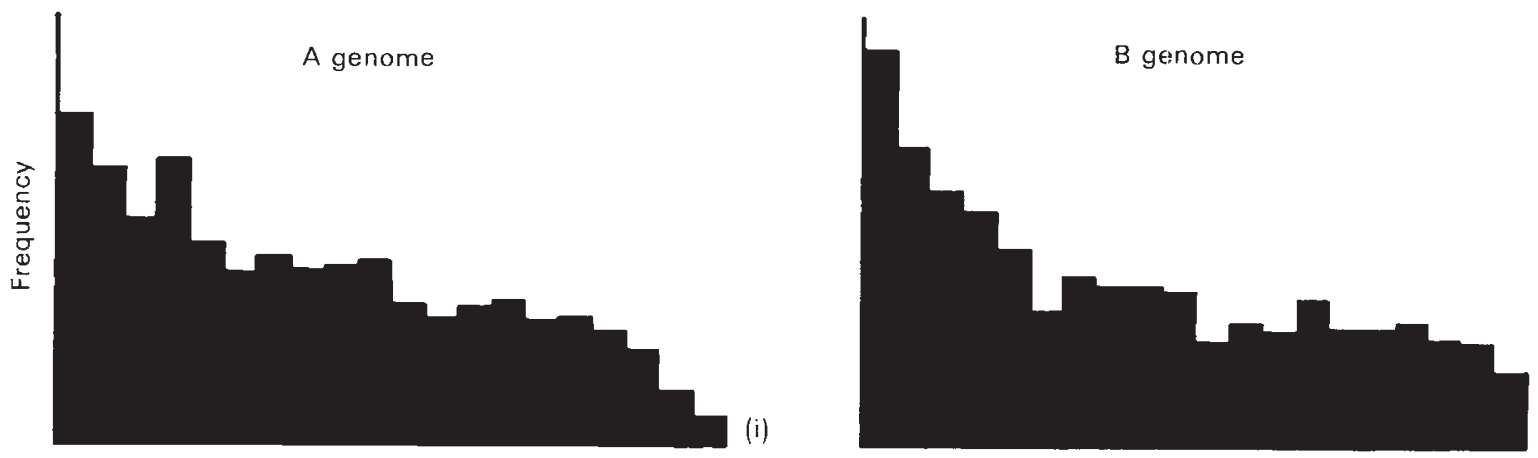

(ii)
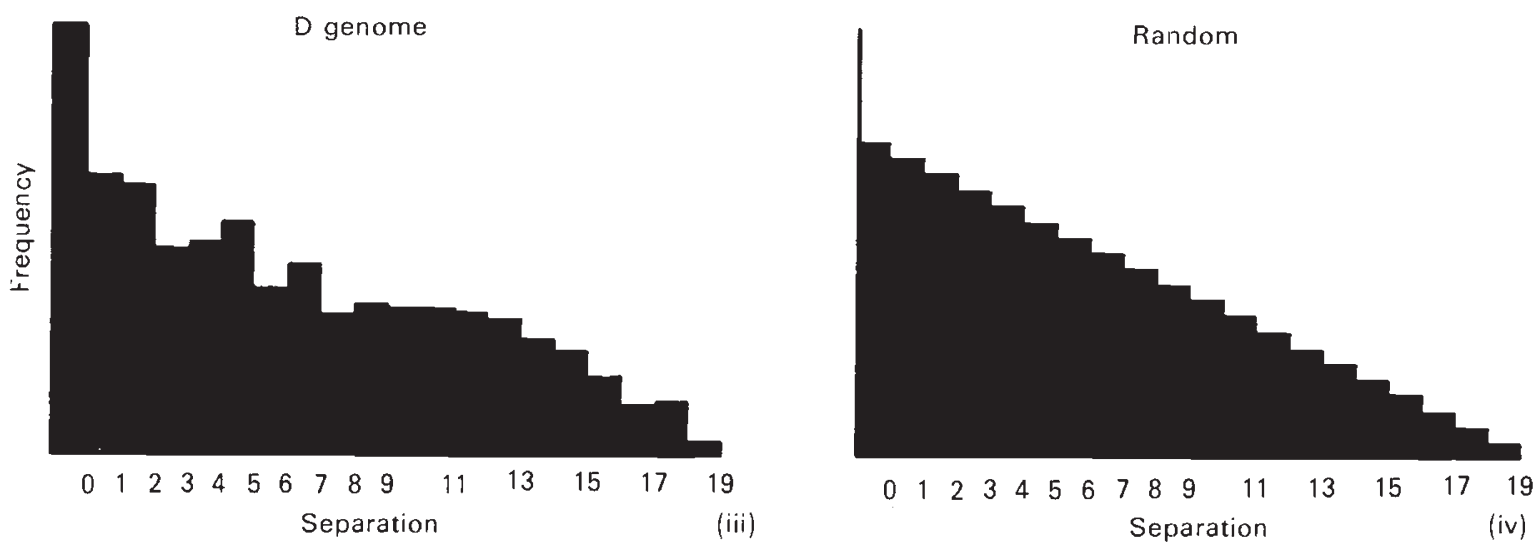

Figure 3 Histograms for each of the three genomes showing the distributions of separations (number of intervening, normal bivalents) of pairs of marked bivalents. (a) $A$ genome, (b) $B$ genome and (c) $D$ genome. (c) shows the expected distribution if the pairs of marked bivalents were distributed at random across the metaphase plate.

(lying together) and with 5, 17, 18 and 19 interveners (table 2 ). The $B$ and $D$ genomes have very highly significant excesses of marked bivalents lying together while the $A$ and $B$ genomes have highly significant excesses of marked bivalents occurring at large separations-from 13 to 19. Another group of results implies that the numbers with 5 interveners may differ from the number of cells with 4 or 6 interveners in the $A$ and $B$ genomes (depleted) and $D$ genome (in excess), although this may be a chance result.

\section{Interaction of methods of scoring}

The histograms shown in figs. 2 and 3 were not independent. There was an interaction between the two methods of scoring so that the results may simply be different measures of the same departure from randomness. As an extreme example, if the marked bivalents always lay at the two end positions on the metaphase plate, then their separation would always be 19 . In the present experiment, it is unknown whether the separation or the positioning of the bivalents was a fixed factor, so analyses were carried out to see (1), if the data for positions accounted for the observed distribution of separations, and, (2), if the data for separations accounted for the observed distribution of positions.

For the first comparison, an expected frequency distribution of separations of marked bivalents was generated, based on the actual distribution of positions of the marked bivalents (as found in the experiment). Fig. 4 shows how one of the random separations, used to make the frequency distribution based on positions, was found. Two random numbers lying in the range from 1 , up to the number of marked bivalents scored in a genome, were generated. Each random number was then compared with the cumulative frequencies of occurrence of marked bivalents (solid black in fig. 4) from one side of the plate to the other (from position 1 to 21 ). The position of the bivalent equivalent to the randomly generated number was 
Table 2 Comparison of the separations of marked bivalents with the random expectation and the expectation based on the positions shown in table 1. (Abbreviations as in table 1 except $E P=$ Expectation based on Positions.)

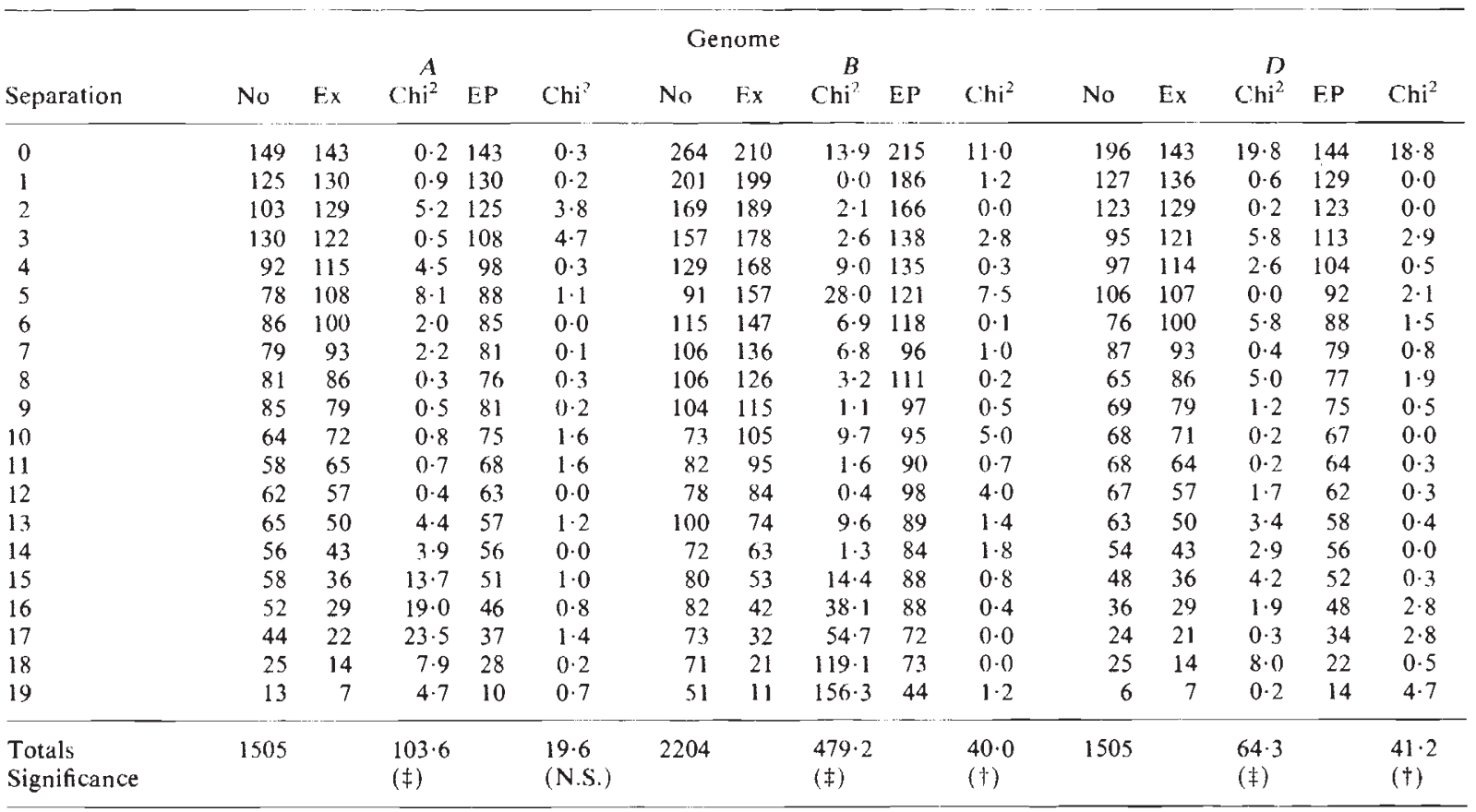

taken to be the number of the left-most position where the cumulative frequency of marked bivalents equalled or exceeded the random number. (If the positions of the two randomly chosen bivalents were the same-not possible in a celltwo new numbers were generated.) The separation (between 0 and 19) of the two random bivalent positions (each between 1 and 21) was then calculated. The selection of two random numbers and generation of a separation was repeated 10,000 times for each of the three genomes. The frequency distribution of these 10,000 separations, between 0 and 19 , was then used as the expectation and compared with the actual distribution of separations of bivalents, using $\mathrm{Chi}^{2}$ tests.

For the second comparison, an expected frequency of positions based on the actual distribution of separations was generated for comparison with the actual distribution of positions, using a similar technique, but using 100,000 randomly chosen separations, rather than positions, in each genome.

\section{Separations derived from positions}

These results (table 2) showed that the positions of the marked bivalents could account for much of the distribution of separations found. The values of $\mathrm{Chi}^{2}$ were considerably reduced from the comparison with a random distribution (19.6 (N.S.), $40 \cdot 0(\dagger)$ and $41 \cdot 2(\dagger)$ for the $A, B$ and $D$ genomes, respectively; reduced from $103 \cdot 6,479 \cdot 2$ and $64 \cdot 3$; $\left.\mathrm{Chi}^{2}(19,0 \cdot 05)=30 \cdot 1\right)$.

In the $D$ genome, more than half of the $\mathrm{Chi}^{2}$ value was accounted for by significantly more pairs of marked bivalents lying together $\left(\mathrm{Chi}^{2}=18 \cdot 8\right.$, $(\$)$ ) than expected and fewer lying at opposite ends of the plate (19 apart, $\left.\mathrm{Chi}^{2}=4 \cdot 7,\left(^{*}\right)\right)$. Similarly, in the $B$ genome, the overall difference was not significant without the 0 separation class, where more bivalents lay together $\left(\mathrm{Chi}^{2}=11 \cdot 0\right.$, $(\ddagger))$ than expected. Individually, the separations of 5 and 10 were also significantly different $\left(\mathrm{Chi}^{2}=\right.$ 7.5 and 5.0, ( $\dagger$ and ${ }^{*}$ respectively)). In the $A$ genome, the distribution was not significantly different from expectation overall, but the separation of 3 was significantly different from the expectation $\left(\mathrm{Chi}^{2}=4.7,\left(^{*}\right)\right)$.

\section{Positions derived from separations}

These expectations (data not shown) were all highly significantly different from the actual results, unlike the results in the previous subsection. Overall $\mathrm{Chi}^{2}$ (10 d.o.f.) values were $60 \cdot 3$ (\$), $322 \cdot 1$ (†) and $77 \cdot 2(\ddagger)$. Within all three 


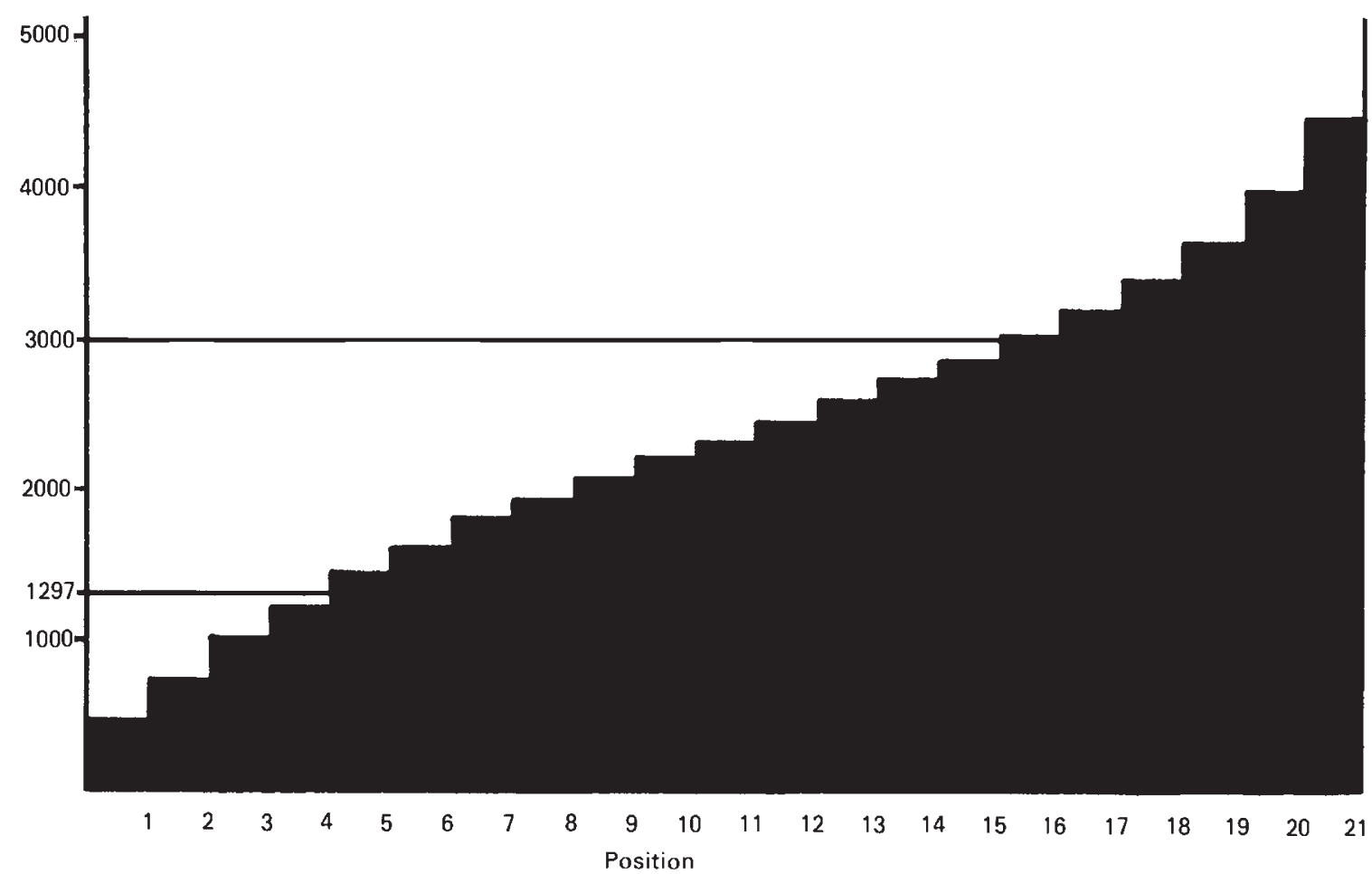

Figure 4 The cumulative frequency of positions of $B$ genome chromosomes. An example to show how random pairs of bivalents were chosen to find the expectation of the distribution of separations of marked pairs of bivalents (based on the distribution of the positions of marked bivalents) is also given. In this example, the random numbers chosen, indicated by the long horizontal lines, 1297 and 3024 (within the range 1 to 4408 ) give positions of 5 and 16, which have a separation of 10 . The separation of 10 was then used as one of the 10,000 values for generating the distribution of separations based on the distribution of positions.

genomes, the actual number of bivalents at the outer positions tended to be significantly higher than expected, and the number near the centre was lower than expected.

\section{DISCUSSION AND CONCLUSIONS}

The experiment reported above has shown various interactions between three effects which all might influence the position of the heteromorphic bivalents on the metaphase plate:

(1) The spreading technique which could affect the marked bivalents differently from the normal bivalents.

(2) The positioning of the marked bivalents within the nucleus, which could be special either because the bivalents were marked or because of the genetical identity of the bivalent.

(3) The interaction of the two marked bivalents with respect to each other in space in the nucleus, which again could relate to their being marked or to the genetic identity of the two bivalents involved.
It is impossible completely and rigorously to separate the effects of the three influences on these data, although some attempt can be made.

\section{Tendency for marked bivalents to lie at the end of the plate}

It is hard to explain why the marked bivalents tend to go to the end of the plate. Clearly, it is impossible for all 21 normal bivalents to tend to lie towards the end position in vivo, so the effect must be due either to redistribution of some or all of the heteromorphic bivalents during spreading, or to the heteromorphs being in special positions in the cell ( 1 or 2 in the previous section). 1t was impossible to distinguish between these two possibilities using squashed preparations. However, the present results clearly show that any analysis of spatial relationships between marked bivalents cannot easily be extended with validity to the behaviour of whole normal bivalents in hexaploid wheat.

Optical examination of unsquashed metaphases would be unlikely to give enough resolution to allow identification of the heteromorphic 
bivalent in most cells. Analysis of serial sections of metaphases, a technique which we have used extensively to examine the undisturbed positions of metaphase chromosomes (e.g., HeslopHarrison and Bennett, 1983a, b), would allow the position as in vivo of marked bivalents to be found, although an impractically large number of cells might need to be reconstructed to allow statistically meaningful comparison of the positions of all the different marked bivalents.

The results from the tests of interaction show that the non-random distribution of separations does not account for the strong tendency for marked bivalents to lie at the end positions. Thus, one or both of the marked bivalents of any pair must tend to lie closer to the end than expected by chance alone.

Kempanna (1963) looked at 50 cells from each of three crosses including one marked bivalent$1 A, 7 B$ and $1 D$. These were not significantly different from random when pooled (Kempanna and Riley, 1964, table 1), and the pooled data, showing excesses of marked bivalents at positions 1, 2, 4 and 9 , were significantly different from those found here for the $B$ genome $\left(\mathrm{Chi}^{2}=24.9,(\dagger)\right)$ but not the $A$ and $D$ genomes $\left(\mathrm{Chi}^{2}=7.53\right.$ and 8.58 respectively, N.S.), although their data were closer to random $\left(\mathrm{Chi}^{2}=3 \cdot 75\right)$. However, when the results for the three genomes (from Kempanna (1963, table 6-1)) were compared individually with the present results there were no significant differences $\left(\mathrm{Chi}^{2}=11 \cdot 8,10.8\right.$ and 10.5 for $A, B$ and $D$ genomes respectively, $0 \cdot 5>P>0 \cdot 1$ ), although the samples were small. Thus, the earlier evidence is not inconsistent with any marked bivalent tending to lie in, or near, end positions in spreads of bivalents at metaphase $\mathrm{I}$.

\section{Interaction of marked bivalents}

There was an interaction in the relative positioning of the bivalents, as shown by the non-random distribution of the number of intervening bivalents. This was largely accounted for by the positions of marked bivalents which were found. However, there was also a tendency for more bivalents than expected to lie together in the $B$ genome (264 compared to an expectation of 215 based on the positions, or 210 if randomly positioned) and $D$ genome ( 196 compared to 144 or 143). This implies that there was some attraction or affinity between the pairs of marked bivalents. The tendency for bivalents to lie at the ends of the plate almost entirely accounts for the increased number of pairs lying at high $(17,18$ or 19$)$ separations which is shown in table 2 , although the number at 19 separation in the $D$ genome was significantly lower than expected based on the data for positions.

When compared with the random expectation, all the genomes were highly significantly different, with generally higher numbers of bivalents at low and high separations than would be expected by chance. This was perhaps because of the tendency of marked bivalents to lie at the ends of the plate, although it was not possible to distinguish between "cause" and "effect" in the present experiment.

\section{Effects of squashing-relationship to actual bivalent position-shape of cell}

In the present work, it is unknown whether the shape of the cell, and/or the metaphase plate, before flattening, influences the positioning of the metaphase bivalents as seen in the spread preparations. It would be necessary to use reconstructions of serially sectioned electron micrographs to see whether the positions of the marked bivalents showed any correlation with the shape of the metaphase plate or cell as discussed above.

Rickards (1984) considered the possibility that the position and orientation of a quadrivalent in Allium was correlated with the geometry of the cell before flattening, but concluded that this was an unlikely cause of his results. He also discussed the possible movements of bivalents during the squashing procedure used to make linear arrays of bivalents. If squashing is applied parallel to the plane of a circular metaphase plate (giving an equatorial squash), then bivalents located at or near the centre of the plate will appear at or near the centre of the spread. Peripheral bivalents may appear at any position, from central to the end, depending upon the direction of squashing with respect to the shape of the metaphase plate. Fig. 5 shows how a bivalent on the periphery of a circular metaphase plate would lie in the end 5 per cent of the length of the squashed plate in more than 20 per cent of linear squashes and in the central 5 per cent less than 3.2 per cent of the time. The situation is different if the metaphase plate, and/or the surrounding section of the cell, is elliptical since these plates will probably tend to be squashed so that the long axis of the original plate and that of the spread bivalents are the same. Thus it seems likely that bivalents originally appearing at the ends of an elliptical in vivo plate (i.e., near the extremes of the major axis of the ellipse) will remain at the ends in the spread, and all other positions will reflect the actual positions of the marked bivalents. 


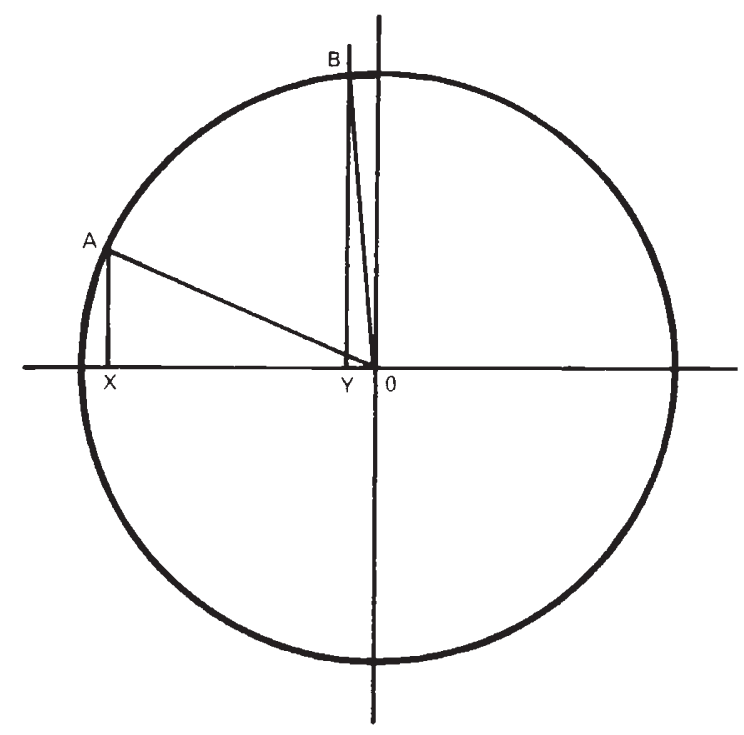

Figure 5 The effect of squashing on the positions of peripheral bivalents, showing that bivalents lying on the periphery of the metaphase plate may be expected to lie in "end" positions much more frequently than at central positions, regardless of the direction of squashing. Bivalents can lie anywhere within the circle shown, which represents the metaphase plate, but when squashed they lie on the horizontal line. The angle XOA is $18 \cdot 2^{\circ}$, where OX is 95 per cent of the radius. Thus bivalents lying on the periphery of the circle will be squashed to lie in the outer 10 per cent of the length of the total metaphase plate in some 20 per cent $(18 \cdot 2 \times 4 / 360 \times 100)$ of squashes. The angle YOB is $2 \cdot 87^{\circ}$, where the line OY is 5 per cent of the radius, so bivalents lying in the inner 10 per cent of the total length of the horizontal line come from bivalents lying on only about 3 per cent of the perimeter.

Centromere sizes are known to vary between chromosomes and bivalents in wheat and other species (Jenkins and Bennett, 1981; Bennett et al., 1981; Heslop-Harrison, 1983). Bivalents with smaller centromeres might tend to be delayed in congression and/or to lie in different places on the metaphase plate. Certainly, some chromosomes generally congress before others in various species (e.g., Vig, 1983). Results with intergeneric hybrids (Bennett, 1983; Finch and Bennett, 1983) have shown that one genome (set of chromosomes) has centromeres which are much less expressed (both by being smaller and having only approximately 10 per cent as many microtubules attached) than those of the other genome. In hexaploid wheat, the bivalents of different genomes may have varying sizes or activities of centromeres-which vary slightly between different chromosomes or bivalents within a genome, and more between genomes-and hence congress at different rates and times, or to different positions, onto the metaphase plate. Jenkins and Bennett (1981) suggested that centromere volumes and chromosome volumes were closely correlated, based on evidence from Festuca nuclei, at least within genomes in single cells. They also suggested that some telocentic chromosomes may have smaller centromeres, in part because of their size. If so, then heteromorphic bivalents may behave differently from other bivalents of the same genome because of the smaller and presumably less active centromeres of their telocentric partner.

Kempanna and Riley (1964) concluded that there was no significant tendency for the positions of marked bivalents to be disturbed because they were rod shaped. However, Rickards (1984) has shown that an interchange quadrivalent at metaphase $\mathrm{I}$ in an Allium species appeared in both particular positions (marginal) and particular orientations ("alternate" if marginal) more frequently than might be expected by random chance alone.

\section{Size of bivalents}

Kempanna and Riley (1964) also concluded that the non-random distribution of bivalents which they found on the wheat metaphase plate was not dependent on the sizes of the bivalents involved, a conclusion which the present data support. The chromosomes of the $B$ genome are generally larger than many of those in the $A$ genome and most in the $D$ genome (Sears, 1954; Giorgi and Bozzini, $1969 ; 1970$; Nishikawa, 1970). It is possible that large and small bivalents might behave differently under the influence of squashing. For example, Heilborn (1936) suggested that secondary association was a differential grouping of the chromosomes of different size and mass under the forces of nuclear division. However, in the present experiment an explanation based on size differences between heteromorphic and normal bivalents is unlikely to be true because the large heteromorphic bivalents-such as $5 B$ or $3 B$-are larger than some of the smaller normal bivalents, such as some of those of the $D$ genome, but still show the tendency to lie at the end positions. Conversely, chromosome $1 D$ is probably the smallest bivalent (Sears, 1954), and when present as a heteromorphic bivalent, the pair is even more likely to be the smallest bivalent, since an arm is missing. However, its behaviour was not significantly different from most of the other bivalents in the same genome (data in Heslop-Harrison, 1983). These results show that size alone does not affect bivalent 
positioning. They agree with the results of Rickards (1984, quoted above) who examined the positioning of abnormal metaphase I quadrivalents in squashes of Allium nuclei, and showed that small and large bivalents were neither found preferentially in marginal nor central positions in flattened metaphases.

It is unlikely that the squashed metaphase plates reflect the actual positions of the marked bivalents in vivo very accurately. Even if the marked bivalents tended actually to be at peripheral positions in the cells, the results from the $B$ genome seem to show they lie at the end position more frequently than would be expected given a random direction of squashing (although a tendency to lie at the ends of the major axis of an ellipse could explain this finding). It is also difficult to explain the tendency of the marked bivalents to lie not at, but near to, the end of the squashed plate in the $A$ and $D$ genome results. Therefore, we conclude that it is probable that the actual positions of the marked bivalents in the cell affect the positions in the squashed plate, but that the physical processes of squashing a pair of marked, heteromorphic, bivalents also affects the positioning of the two bivalents.

\section{Different behaviour of the three diploid genomes}

The genome $(A, B$ or $D)$ has a large effect on both the positioning and separations of marked bivalents (seen in both fig. 2 and fig. 3). As discussed above, the basis of the difference is unlikely to be bivalent size, nor the effect of being marked since chromosomes from all genomes were marked and treated in similar ways in the experiment. Thus some other, possibly genetical control of relative or absolute bivalent position may be acting differently on the three genomes. The physical distortion caused by the marking might have different effects on each genome or alternatively positions of the three genomes could be different, and affect the bivalent positioning differently in each genome.

\section{Evidence for a fixed disposition of genomes}

An explanation of the differences between the genomes could involve differences in their relative spatial separation. If the three genomes are in part concentrically spatially separated, the $B$ genome could have more opportunity for its chromosomes to move to the actual end of the plate than the others. This possibility is particularly interesting in view of results of recent studies of intergeneric and interspecific grass hybrids which have shown that the chromosomes of the two parental genomes tend to be separated concentrically-i.e., one set of chromosomes tending to be peripheral to another (see Finch et al., 1981; Linde-Laursen and Jensen, 1984; Bennett, 1985). This work was carried out on diploid hybrids, but results (Bennett and Smith, unpublished) for autotetraploid, Hordeum vulgare $\times$ Secale africanum, also show concentric separation of parental sets exactly as found in similar hybrids at the diploid level. Perhaps the three genomes of hexaploid wheat behave similarly with the outer, peripheral, $B$ genome, tending to surround the more central $A$ and $D$ genomes. This concept of the genomes lying concentrically may also be supported by the results of Avivi et al. $(1982 a)$, in somatic cells, who stated that "within each genome, the homologous chromosomes were significantly closer to one another than were nonhomologues" and that $(1982 b)$ "the three wheat genomes are not intermixed but, rather, tend to occupy different areas of the somatic nucleus".

In hexaploid wheat, the three genomes might be concentrically separated with the different genomes occupying different regions of the nucleus. The individual genomes, although interacting, could all behave differently and may be acting independently. Further experiments, involving both serially-sectioned normal and marked karyotypes, as well as this type of squash experiment, will be required to test such theories.

Acknowledgements J.S. H.-H. thanks Peterhouse, Cambridge for the award of a William Stone Research Fellowship.

\section{REFERENCES}

AVIVI, L., FELDMAN, M. AND BROWN, M. 1982a. An ordered arrangement of chromosomes in the somatic nucleus of common wheat, Triticum aestivum L. I. Spatial relationships between chromosomes of the same genome. Chromosoma, 86, 1-16.

AVIVI, I., FELIDMAN, M. AND BROWN, M. 1982b. An ordered arrangement of chromosomes in the somatic nucleus of common wheat, Triticum aestivum L. 1I. Spatial relationships between chromosomes of the different genomes. Chromosoma, 86, 17-26.

BENNETT, M. D. 1983. The spatial distribution of chromosomes. In: Kew Chromosome Conference II, pp. 71-79, eds. P. E. Brandham and M. D. Bennett. George Allen and Unwin, London.

BENNFIT, M. D. 1985. Nuclear architecture and its manipulation. In: 16th Stadler Genetics Symposium on "Gene manipulation in plant improvement", ed. J. P. Gustafson. Plenum, New York, pp. 469-502.

BENNI:TT, M. D., SMITH, J. R., WARD, J. ANI JENKINS, G. 1981 The relationship between nuclear DNA content and centromere volume in higher plants. J. Cell Sci., 47, 91-115. 
CHAPMAN, V. ANI RILEY, R. 1966. The allocation of the chromosomes of Triticum aestivum to the $A$ and $B$ genomes and evidence on genome structure. Can. J. Genet. Cytol., $8,57-63$.

DARlington, C. D. 1937. Recent Advances in Cytology, J. \& A. Churchill, London.

DARLington, C. D. AND MOFFETT, A. A. 1930. Primary and secondary chromosome balance in Pyrus. J. Genet., 22, $129-151$

DVORAK, J. 1983. The origin of wheat chromosomes $4 A$ and $4 B$ and their genome reallocation. Can. J. Genet. Cytol. $25,210-214$.

FELIMMAN, M. AND AVIVI, L. 1973. Non-random association and meiotic pairing in common wheat. Proc. 3rd Int. Wheat Genet. Symp. pp. 31-40.

FINCH, R. A. AND BENNETT, M. D. 1983. The mechanism of somatic chromosomes elimination in Hordeum. In: Kew Chromosome Conference II, pp. 147-154, eds. P. E. Brandham and M. D. Bennett. George Allen \& Unwin, London.

FINCH, R. A., SMITH, J. B. AND BENNETT, M. D. 1981. Hordeum and Secale genomes lie apart in a hybrid. J. Cell Sci., 52, 391-403.

GIORGI, B, AND BOZZINI, A. 1969. Karyotype analysis in Triticum. III. Analysis of the presumed progenitors of polyploid wheats. Caryologia, 22, 279-287.

GIORGI, B. ANI BOZZINI, A. 1970. Karyotype analysis in Triticum. V. Identification of chromosomes of bread and Durum wheats using aneuploids of Chinese Spring. Caryologia, 23, 565-574.

GUSTAFSSON, A. 1934. Primary and secondary association in Taraxacum. Hereditas, 20,1-31.

HESLOP.HARRISON, J. S. 1983. Spatial disposition of chromosomes in wheat species. Ph.D. University of Cambridge.

HESLOP-HARRISON, J. S. AND BENNETT, M. D. $1983 a$. Prediction and analysis of spatial order in haploid chromosome complements. Proc. roy. Soc. Lond. B., 218, 211-223.

HESLOP-HARRISON, J. S. AND BENNETT, M. D. $1983 \mathrm{~b}$. The spatial order of chromosomes in root tip metaphases of Aegilops umbellulata. Proc. roy. Soc. Lond. B., 218, 225-239.
HEILBORN, O. 1936. The mechanics of so-called secondary association between chromosomes. Hereditas, 22, 167-188.

JENKINS, G. AND BENNETT, M. D. 1981. The intranuclear relationship between centromere volume and chromosome size in Festuca scariosa $\times$ drymeja. J. Cell Sci., 47, 117-125.

KEMPANNA, C. 1963. Investigations into the genetic regulation of meiotic chromosome behaviour in Triticum aestivum $\mathrm{L}$. Ph.D. University of Cambridge.

KEMPANNA, C. AND RILEY, R. 1964. Secondary association between genetically equivalent bivalents. Heredity, 19, 289299.

LINDE-LAURSEN, I. AND JENSEN, J. 1984. Separate location of parental chromosomes in squashed metaphases of hybrids between Hordeum vulgare L. and four polyploid, alien species. Hereditas, 100, 67-73.

NANDI, H. K. 1936. The chromosome morphology, secondary association and origin of cultivated rice. J. Genet., 33, 315-336.

NISHIKAWA, K. 1970. DNA content of the individual chromosomes and genomes in wheat and its relatives. Seiken Ziho, $22,57-65$.

OKAMOTO, M. 1962. Identification of the chromosomes of common wheat belonging to the $A$ and $B$ genomes. Can. $J$. Genet. Cytol., 4, 31-37.

RICKARDS, G. K. 1984. Position and orientation in the metaphase equator of an interchange quadrivalent of Allium triquetrum. Genet. Res. Camb., 43, 139-148.

RILEY, R. 1960. The secondary pairing of bivalents with genetically similar chromosomes. Nature, 185, 751-752.

SEARS, E. R. 1952. Homoeologous chromosomes in Triticum aestivum. Genetics, 37, 624-625.

SEARS, E. R. 1954. The aneuploids of common wheat. Res. Commun. 572, University of Missouri College of Agriculture.

SEARS, E. R. 1959. The aneuploids of common wheat. Proc. 1st. Int. Wheat Genet. Symp. pp. 221-228.

VIG, B. K. 1983. Sequence of centromere separation: occurrence, possible significance and control. Cancer Genet. \& Cytogenet., 8, 249-274.

Note added in Proof: Yacobi et al. $(1985 a, b)$ have recently reported finding that linearly arranged metaphase I spreads of similar hybrid lines to those used in the present paper do not represent the presquashed arrangement of chromosomes as faithfully as circularly arranged metaphases. Their data, on circularly arranged metaphases, showed "greater intimacy between bivalents of the same genome than between bivalents of different genomes" which may support the evidence for independence of the genomes presented above.

Yacobi, Y. Z., Levanony, H. and Feldman, M. 1985a. An ordered arrangement of bivalents at first meiotic metaphase of wheat. I. Hexaploid wheat. Chromosoma, 91, 347-354.
Yacobi, Y. Z., Levanony, H. and Feldman, M. 1985b. An ordered arrangement of bivalents at first meiotic metaphase of wheat. II. Tetraploid wheat. Chromosoma, 91, 355-358. 